\title{
Erratum for: Primary cultures of mouse small intestinal epithelial cells using the dissociating enzyme type I collagenase and hyaluronidase
}

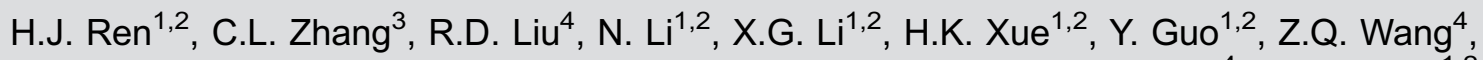 \\ J. Cui ${ }^{4}$ and L. Ming ${ }^{1,2}$ \\ ${ }^{1}$ Department of Clinical Laboratory, the First Affiliated Hospital of Zhengzhou University, Zhengzhou, Henan, China \\ ${ }^{2}$ Key Clinical Laboratory of Henan Province, Zhengzhou, Henan, China \\ ${ }^{3}$ Department of General Surgery, the People's Hospital of Zhengzhou, Zhengzhou, Henan, China \\ ${ }^{4}$ Department of Parasitology, Medical College, Zhengzhou University, Zhengzhou, Henan, China
}

Erratum for: Braz J Med Biol Res | doi: 10.1590/1414-431X20175831

The Journal would like to correct the order of the authors for correspondence in the article "Primary cultures of mouse small intestinal epithelial cells using the dissociating enzyme type I collagenase and hyaluronidase" that was published incorrectly in volume 50 no. 5 (2017) in the Brazilian Journal of Medical and Biological Research <http://dx.doi.org/ 10.1590/1414-431X20175831>

The correct order of the authors for correspondence is:

Correspondence: L. Ming: <mingliang_2015@sina.com > | J. Cui: <cuij@zzu.edu.cn> 